\title{
A NOTE ON THE TRACE THEOREM FOR BESOV-TYPE SPACES OF GENERALIZED SMOOTHNESS ON $d$-SETS
}

\author{
VANJA WAGNER \\ University of Zagreb, Croatia
}

\begin{abstract}
The main goal of this paper is to give a complete proof of the trace theorem for Besov-type spaces of generalized smoothness associated with complete Bernstein functions satisfying certain scaling conditions on $d$-sets $D \subset \mathbb{R}^{n}, d \leq n$. The proof closely follows the classical approach by Jonsson, Wallin in [18] and the trace theorem for classical Besov spaces. Here, the trace space is defined by means of differences. When $d=n$, as an application of the trace theorem, we give a condition under which the test functions $C_{c}^{\infty}(D)$ are dense in the trace space on $D$.
\end{abstract}

\section{INTRODUCTION}

Besov-type spaces of generalized smoothness were introduced in the seventies by M.L. Goldman and G.A. Kalyabin as a generalization of the classical Sobolev and Besov spaces. Since then they have been studied by many authors from various points of view. Due to their close connection to the theory of stochastic processes and probability theory, these spaces continue to be of further interest. For a unified and general approach to Besov spaces and function spaces of generalized smoothness and a review of results we refer the reader to $[5,6,16]$.

In this paper we consider traces on $d$-sets of a special class of Besov spaces of generalized smoothness associated with certain continuous negative definite functions. Let $\nu:(0, \infty) \rightarrow(0, \infty)$ be a non-increasing function satisfying

$$
\nu(r) \leq c_{1} \nu(r+1) \text { and } \int_{0}^{\infty}\left(1 \wedge s^{2}\right) \nu(s) s^{n-1} d s<\infty .
$$

2010 Mathematics Subject Classification. 46E35, 60J75, 60G51.

Key words and phrases. Function spaces of generalized smoothness, $d$-sets, trace space, Bernstein functions. 
for all $r \geq 1$ and some constant $c_{1}>0$. Such a function $\nu$ is a radial Lévy density of an isotropic unimodal Lévy process with characteristic exponent

$$
\psi(|\xi|)=\int_{\mathbb{R}^{n} \backslash\{0\}}(1-\cos (x \cdot \xi)) \nu(|x|) d x, \xi \in \mathbb{R}^{n} .
$$

Throughout the paper we will assume that $\psi$ satisfies one or both of the following scaling conditions,

(H1): there exist constants $0<\delta_{1} \leq \delta_{2}<1$ and $a_{1}, a_{2}>0$ such that

$$
a_{1} \lambda^{2 \delta_{1}} \psi(t) \leq \psi(\lambda t) \leq a_{2} \lambda^{2 \delta_{2}} \psi(t), \quad \lambda \geq 1, t \geq 1
$$

(H2): there exist constants $0<\delta_{3} \leq \delta_{4}<1$ and $a_{3}, a_{4}>0$ such that

$$
a_{3} \lambda^{2 \delta_{3}} \psi(t) \leq \psi(\lambda t) \leq a_{4} \lambda^{2 \delta_{4}} \psi(t), \quad \lambda \geq 1, t<1 .
$$

Under condition (H1), by $[19,(2.1),(2.2)]$, there exists a complete Bernstein function $\phi$ and a constant $\gamma_{2} \geq 1$ such that

$$
\gamma_{2}^{-1} \phi\left(|\xi|^{2}\right) \leq \psi(|\xi|) \leq \gamma_{2} \phi\left(|\xi|^{2}\right), \xi \in \mathbb{R}^{n},
$$

and the radial Lévy density $\nu$ enjoys the following property: for every $R>0$

$$
\nu(r) \asymp \frac{\phi\left(r^{-2}\right)}{r^{n}}, r \in(0, R) .
$$

Due to the equivalence of norms, we will always assume that the constant $\gamma_{2}$ in (1.3) is equal to 1. Furthermore, by [19, Lemma 2.1] every Bernstein function $\phi$ satisfies the following useful inequality,

$$
1 \wedge \lambda \leq \frac{\phi(\lambda r)}{\phi(r)} \leq 1 \vee \lambda, \quad \lambda, r>0
$$

For further results on Bernstein functions, we refer the reader to [22]. Define by

$$
H^{\psi, 1}\left(\mathbb{R}^{n}\right)=\left\{u \in L^{2}\left(\mathbb{R}^{n}\right):\|u\|_{\psi, 1}:=\int_{\mathbb{R}^{n}}(1+\psi(\xi))|\mathcal{F} u(\xi)|^{2} d \xi<\infty\right\}
$$

the $\psi$-Bessel-type potential space on $\mathbb{R}^{n}$. These spaces naturally arise as domains of Dirichlet forms associated with Lévy processes with the characteristic exponent $\psi$. They were introduced by N. Jacob in seminal papers $[11,12]$, where the author studied the construction of Feller processes starting from the corresponding symbol. A thourough investigation of these spaces can be found in the following works $[5,8,9,13,14,15,16]$. In the first chapter, we recall basic definitions and results regarding Besov-type spaces of generalized smoothness, connect them with $\psi$-Bessel-type potential spaces and provide several characterizations of the corresponding norms, based on differences.

Traces of Sobolev spaces on bounded domains with fractal boundary were initially studied in [27] and later this result was generalized in [7] for fractional Sobolev spaces. In the latter, the authors applied the trace results in studying Dirichlet forms related to subordinate reflecting diffusions (with stable 
subordinator) in non-smooth domains. As mentioned before, in this paper we consider traces on $d$-sets of $\psi$-Bessel-type potential spaces and their representation via differences. This approach allows us to easily associate these spaces with domains of Dirichlet forms corresponding to a certain class of purely discontinuous symmetric Markov processes. A trace theorem on $h$-sets for generalized Besov-type spaces associated with functions satisfying slightly different assumptions was presented by Knopova and Zähle in [20]. The authors consider the quarkonial representations of the trace spaces, as well as the representation via differences, and prove their equivalence. In the following paper [28], Zähle proves a potential representation of the trace spaces and the corresponding trace Dirichlet forms. In order to make the theory of generalized Besov spaces more approachable in the context of application to the theory of stochastic processes, we present a complete and detailed proof of the trace theorem based on the characterization via differences, following the approach by Jonsson and Wallin in [18] for classical Besov spaces. In order to obtain the trace theorem, we use several representation results and interpolation theorems, which are direct generalizations of the corresponding results for classical Besov spaces from [18, 24, 25].

In the following two chapters, we give a complete proof of the trace theorem for spaces $H^{\psi, \alpha}\left(\mathbb{R}^{n}\right)$ (see Definition 2.2) on $d$-sets, where the trace space is defined by means of differences.

Definition 1.1. Let $D$ be a non-empty Borel subset of $\mathbb{R}^{n}$ and $0<d \leq n$. A positive Borel measure $\mu$ on $D$ is called a d-measure if there exist positive constants $c_{1}$ and $c_{2}$ such that for all $x \in D$ and $r \in(0,1]$,

$$
c_{1} r^{d} \leq \mu(B(x, r)) \leq c_{2} r^{d} .
$$

A non-empty Borel set $D$ is called a d-set if there exists a d-measure $\mu$ on $D$.

Note that, by definition, all $d$-measures on $D$ are equivalent to the restriction of the $d$-dimensional Hausdorff measure to $D$. For a $d$-set $D$ in $\mathbb{R}^{n}$ with $d$-measure $\mu$, we define the trace space as

$$
\begin{aligned}
& H^{\psi, 1}(D, \mu)=\left\{u \in L^{2}(D, \mu):\|u\|_{(1), D, \mu}<\infty\right\}, \\
& \|u\|_{(1), D, \mu}:=\|u\|_{L^{2}(D, \mu)}+\left(\iint_{|x-y|<1}|u(x)-u(y)|^{2} \frac{\phi\left(|x-y|^{-2}\right)}{|x-y|^{2 d-n}} \mu(d x) \mu(d y)\right)^{\frac{1}{2}} .
\end{aligned}
$$

Following the classical approach from [18], the proof of the trace theorem is divided into three parts; the restriction theorem, the extension theorem for $d<n$ and finally, the extension theorem when $d=n$. The following theorem is a direct consequence of the main results of this paper, i.e. Theorem 3.2, Theorem 4.2 and Theorem 4.4 for traces of spaces $H^{\psi, \alpha}\left(\mathbb{R}^{n}\right)$ on $d$-sets in $\mathbb{R}^{n}$. 
THEOREM 1.2. Let $D$ be a $n$-set in $\mathbb{R}^{n}$ and $\psi$ a radial function defined by (1.2) such that $(\mathrm{H} 1)$ and $(\mathrm{H} 2)$ hold. There exists a continuous

(i) restriction operator $R: H^{\psi, 1}\left(\mathbb{R}^{n}\right) \rightarrow H^{\psi, 1}(D, \mu)$,

(ii) extension operator $E: H^{\psi, 1}(D, \mu) \rightarrow H^{\psi, 1}\left(\mathbb{R}^{n}\right)$, such that $R E u=u$, $\mu$-a.e. on $D$, for all $u \in H^{\psi, 1}(D, \mu)$.

The trace theorem for $\psi$-Bessel-type potential spaces is motivated by the analysis of the boundary behaviour of the censored symmetric Lévy-type process on a $n$-set $D \subset \mathbb{R}^{n}$ associated with the Dirichlet space $H_{0}^{\psi, 1}(D)$, considered in $[3,26]$. Here $H_{0}^{\psi, 1}(D)$ is the closure of the space $C_{c}^{\infty}(D)$ with respect to the norm $\|\cdot\|_{(1), D, \lambda_{D}}$ and $\lambda_{D}$ is the Lebesgue measure on $D$. By showing that the extended space $H^{\psi, 1}(D):=H^{\psi, 1}\left(D, \lambda_{D}\right)$ is the trace space on a $n$-set $\bar{D}$ of $H^{\psi, 1}\left(\mathbb{R}^{n}\right)$, we can relate the question of the equivalence of spaces $H^{\psi, 1}(D)$ and $H_{0}^{\psi, 1}(D)$ to the capacity of the boundary $\partial D$ with respect to the norm on $H^{\psi, 1}\left(\mathbb{R}^{n}\right)$. An immediate consequence of this result is the characterization of the finite time approach of the censored process on $D$ to the boundary $\partial D$ in terms of the polarity of the boundary with respect to the Lévy-type process in $\mathbb{R}^{n}$ associated with the Dirichlet space $H^{\psi, 1}\left(\mathbb{R}^{n}\right)$, for example see [26, Theorem 1.1, Corollary 2.9]. Here we present this result on the correspondence of spaces $H^{\psi, 1}(D)$ and $H_{0}^{\psi, 1}(D)$ in terms of the Hausdorff dimension of the boundary $\partial D$. For an equivalent result in the case of the classical Bessel potential space see [3, Corollary 2.8].

Corollary 1.3. Suppose that $D \subset \mathbb{R}^{n}$ is an open $n$-set, $\phi$ is a complete Bernstein function such that $(\mathrm{H} 1)$ and $(\mathrm{H} 2)$ hold and $\psi(\xi)=\phi\left(|\xi|^{2}\right)$.

(i) Suppose that $2 \delta_{2} \leq n$ and that $\mathcal{H}_{h}\left(\partial D \cap K_{m}\right)<\infty$ for an increasing sequence of Borel sets $K_{m}$ such that $\cup_{m \in \mathbb{N}} K_{m} \supset \partial D$, where $h(r)=$ $r^{n-2 \delta_{2}}$ if $2 \delta_{2}<n$ and $h(r)=\max \{\log r, 0\}$ when $2 \delta_{2}=n=1$, then $H^{\psi, 1}(D)=H_{0}^{\psi, 1}(D)$.

(ii) If $2\left(\delta_{1} \wedge \delta_{3}\right) \geq n=1$ or $\mathcal{H}^{d}(\partial D)>0$ for some $d>n-2 \delta_{1} \geq 0$ then $H_{0}^{\psi, 1}(D) \subsetneq H^{\psi, 1}(D)$.

For easier notation, we write $f \asymp g$ if there exists a constant $c>1$ such that for all $x, c^{-1} g(x) \leq f(x) \leq c g(x)$. Equivalently, $f \lesssim g$ if there exists a constant $c>0$ such that for all $x, f(x) \leq c g(x)$. By $\lambda$ we denote the Lebesgue measure on $\mathbb{R}^{n}$.

\section{Besov-type SPACES OF GENERALIZED SMOOTHNESS AND EQUivalent NORMS}

The aforementioned $\psi$-Bessel-type potential space is a type of a much more general class of function spaces called Besov-type spaces of generalized smoothness, see $[5,6,16]$. First we recall these spaces in their most general form. 
Definition 2.1. A sequence $\left(\gamma_{j}\right)_{j \in \mathbb{N}_{0}}$ of positive real numbers is called

(i) almost increasing if there exists $d_{0}>0$ such that $d_{0} \gamma_{j} \leq \gamma_{k}$, for all $j \leq k$

(ii) strongly increasing if it is almost increasing and in addition there exists a $\kappa_{0} \in \mathbb{N}$ such that $2 \gamma_{j} \leq \gamma_{k}$, for $j \leq k-\kappa_{0}$

(iii) of bounded growth if there are positive constants $d_{1}$ and $J_{0} \in \mathbb{N}_{0}$ such that $\gamma_{j+1} \leq d_{1} \gamma_{j}$, for all $j \geq J_{0}$

(iv) an admissible sequence if both $\left(\gamma_{j}\right)_{j \in \mathbb{N}_{0}}$ and $\left(\gamma_{j}^{-1}\right)_{j \in \mathbb{N}_{0}}$ are of bounded growth and $J_{0}=0$, i.e. there exist positive constants $d_{0}$ and $d_{1}$ such that $d_{0} \gamma_{j} \leq \gamma_{j+1} \leq d_{1} \gamma_{j}$, for all $j \in \mathbb{N}_{0}$.

Definition 2.2. Let $N=\left(N_{j}\right)_{j \in \mathbb{N}_{0}}$ be a strongly increasing sequence and define

$\Omega_{0}^{N}=\left\{x \in \mathbb{R}^{n}:|x| \leq N_{0}\right\}$ and $\Omega_{j}^{N}=\left\{x \in \mathbb{R}^{n}: N_{j-1} \leq|x| \leq N_{j+1}\right\}, j \in \mathbb{N}$.

Let $\Phi^{N}$ be a collection of all partitions of unity of $C_{c}^{\infty}\left(\mathbb{R}^{n}\right)$ functions associated with this decomposition. Let $\sigma=\left(\sigma_{j}\right)_{j \in \mathbb{N}_{0}}$ be an admissible sequence respectively and $\left(\varphi_{j}^{N}\right)_{j \in \mathbb{N}_{0}} \in \Phi^{N}$. The Besov space of generalized smoothness associated with $N$ and $\sigma$ is defined by

$$
B_{2}^{\sigma, N}=\left\{g \in S^{\prime}\left(\mathbb{R}^{n}\right):\|g\|_{B, \sigma, N}:=\left\|\left(\sigma_{j} \varphi_{j}^{N}(D) g\right)_{j \in \mathbb{N}_{0}} \mid l_{2}\left(L_{2}\left(\mathbb{R}^{n}\right)\right)\right\|<\infty\right\},
$$

where $\varphi(D) g(x)=\mathcal{F}^{-1}(\varphi(\cdot) \mathcal{F} g)(x)$ and

$$
\left\|\left(f_{j}\right)_{j \in \mathbb{N}_{0}} \mid l^{2}\left(L^{2}\left(\mathbb{R}^{n}\right)\right)\right\|=\left(\sum_{j=0}^{\infty}\left\|f_{j}\right\|_{L^{2}\left(\mathbb{R}^{n}\right)}^{2}\right)^{\frac{1}{2}} .
$$

By [5, Remark 10.1.2.] the space $B_{2}^{\sigma, N}$ is independent of the choice of system $\left(\varphi_{j}^{N}\right)_{j \in \mathbb{N}_{0}}$ in the sense of equivalent norms. We will restrict ourselves to a special subclass of spaces $B_{2}^{\sigma, N}$ associated with an admissible symbol.

Definition 2.3. A non-negative function $a \in C^{\infty}\left(\mathbb{R}^{n}\right)$ is an admissible symbol if

(i) $\lim _{|x| \rightarrow \infty} a(x)=\infty$,

(ii) a is almost increasing in $|x|$, i.e. there exist constants $\delta_{0} \geq 1$ and $R>0$ such that $a(x) \leq \delta_{0} a(y)$ if $R \leq|x| \leq|y|$,

(iii) there exists an $m>0$ such that $x \rightarrow \frac{a(x)}{|x|^{m}}$ is almost decreasing in $|x|$,

(iv) for every multi-index $\alpha \in \mathbb{N}_{0}^{n}$ there exist constants $c_{\alpha}>0$ and $R>0$ such that

$$
\left|D^{\alpha} a(x)\right| \leq c_{\alpha} \frac{a(x)}{\left(1+|x|^{2}\right)^{|\alpha| / 2}}, \quad \forall|x| \geq R .
$$

The family of all admissible functions will be denoted by $\mathcal{A}$. 
Note that for every Bernstein function $\phi$ such that $\lim _{r \rightarrow \infty} \phi(r)=\infty$, the function $\xi \rightarrow \phi\left(|\xi|^{2}\right)$ is an admissible symbol, see [6, Lemma 3.1.13].

Remark 2.4. By [6, Lemma 3.1.17, Remark 3.1.18], the sequence $N_{j}^{a, r}=$ $\sup \left\{|x|: a(x) \leq 2^{j r}\right\}, j \in \mathbb{N}_{0}$, where $a \in \mathcal{A}$ and $r>0$, is strongly increasing.

For $a \in \mathcal{A}$ we define the Besov space of generalized smoothness associated with $a$ as $B_{2}^{\sigma, N^{a, 2}}\left(\mathbb{R}^{n}\right)$, where $\sigma=\left\{2^{j}\right\}_{j \in \mathbb{N}_{0}}$ is an admissible sequence. These spaces have two useful representations in the sense of equivalent norms; one given by the Littlewood-Paley-type theorem and the other by means of differences.

Proposition 2.5. [6, Theorem 3.1.20, Corollary 3.1.21] Let $a \in \mathcal{A}, N=$ $N^{a, 2}$ the strongly increasing sequence associated with $a, \alpha>0$ and $\sigma^{\alpha}=$ $\left\{2^{\alpha j}\right\}_{j \in \mathbb{N}_{0}}$ an admissible sequence. Then the space $\left(H^{a, \alpha}\left(\mathbb{R}^{n}\right),\|\cdot\|_{a, \alpha}\right)$, defined by

$$
\begin{aligned}
& H^{a, \alpha}\left(\mathbb{R}^{n}\right)=\left\{u \in L^{2}\left(\mathbb{R}^{n}\right):\|u\|_{a, \alpha}<\infty\right\} \\
& \|u\|_{a, \alpha}:=\left\|(i d+a(D))^{\alpha / 2} u\right\|_{L_{2}\left(\mathbb{R}^{n}\right)}=\left(\int_{\mathbb{R}^{n}}(1+a(\xi))^{\alpha}|\hat{u}(\xi)|^{2} d \xi\right)^{\frac{1}{2}},
\end{aligned}
$$

is equivalent to $\left(B_{2}^{\sigma^{\alpha}, N^{a, 2}}\left(\mathbb{R}^{n}\right),\|\cdot\|_{B, \sigma^{\alpha}, N^{a, 2}}\right)$.

Proposition 2.5 implies that Besov-type spaces of generalized smoothness associated with $\psi$ can be characterized as

$$
H^{\psi, \alpha}\left(\mathbb{R}^{n}\right)=\left\{u \in S^{\prime}\left(\mathbb{R}^{n}\right): \exists f \in L^{2}\left(\mathbb{R}^{n}\right) \text { such that } \mathcal{F} u=(1+\psi)^{-\alpha / 2} \mathcal{F} f\right\} .
$$

Since the function $x \mapsto(1+x)^{-\alpha / 2}$ is completely monotone for every $\alpha>0$, by $\left[22\right.$, Theorem 3.7] the function $(1+\phi)^{-\alpha / 2}$ is also completely monotone. By Schoenberg's theorem, [23, Theorem 2] it follows that the function $(1+\psi)^{-\alpha / 2}$ is a positive definite function and therefore a Fourier transforms of an integrable function, [22, Theorem 4.14], called the Bessel-type potential $K_{\psi, \alpha}$. This means that the space $H^{\psi, \alpha}\left(\mathbb{R}^{n}\right)$ defined by $(2.1)$ can be characterized as a convolution space via the $\psi$-Bessel convolution kernel $K_{\psi, \alpha}$ i.e.

$$
H^{\psi, \alpha}\left(\mathbb{R}^{n}\right)=\left\{K_{\psi, \alpha} * f: f \in L^{2}\left(\mathbb{R}^{n}\right)\right\}, \quad\left\|K_{\psi, \alpha} * f\right\|_{\psi, \alpha}:=\|f\|_{L^{2}\left(\mathbb{R}^{n}\right)} .
$$

For similar calculations we refer the reader to $[8,9]$.

From now on we assume that the function $\psi$ satisfies conditions (H1) and (H2). The following estimates for the kernels $K_{\psi, \alpha}$ were obtained in [20, Remark 33, Remark 34] and [28].

Lemma 2.6. Let $\alpha>0$ and assume ( $\mathrm{H} 1)$ and ( $\mathrm{H} 2)$ hold. If $\alpha\left(\delta_{2} \vee \delta_{4}\right)<n$ then there exist constants $c_{i}=c_{i}(\phi, \alpha, n)>0, i=1,2$, such that for all $x \in \mathbb{R}^{n}$ 
and $0 \leq j \leq n$

$$
\begin{aligned}
\left|K_{\psi, \alpha}(x)\right| & \leq \frac{c_{1}}{|x|^{n} \phi\left(|x|^{-2}\right)^{\alpha / 2}}, \\
\left|\left(K_{\psi, \alpha}(x)\right)_{x_{j}}^{\prime}\right| & \leq \frac{c_{2}}{|x|^{n+1} \phi\left(|x|^{-2}\right)^{\alpha / 2}} .
\end{aligned}
$$

Next, we consider the characterization of spaces $H^{\psi, \alpha}\left(\mathbb{R}^{n}\right)$ via differences. First note that by applying (1.2) and the Parseval's identity (see for example [17, Lemma 3.1] or [10, Example 1.4.1]), it easily follows that

$$
\|u\|_{\psi, 1}=\|u\|_{L^{2}\left(\mathbb{R}^{n}\right)}+\left(\frac{1}{2} \int_{\mathbb{R}^{n}} \int_{\mathbb{R}^{n}}(u(x+y)-u(x))^{2} \nu(y) d y d x\right)^{\frac{1}{2}}
$$

for all $u \in H^{\psi, 1}\left(\mathbb{R}^{n}\right)$. We also introduce an equivalent norm on $H^{\psi, 1}\left(\mathbb{R}^{n}\right)$, which we later use in the proof of the trace theorem,

$$
\|u\|_{(1)}=\|u\|_{L^{2}\left(\mathbb{R}^{n}\right)}+\left(\iint_{|x-y|<1}|u(x)-u(y)|^{2} \frac{\phi\left(|x-y|^{-2}\right)}{|x-y|^{n}} d x d y\right)^{\frac{1}{2}},
$$

with the equivalence of norms $\|\cdot\|_{(1)}$ and $\|\cdot\|_{\psi, 1}$ following from (2.3), (1.4) and the fact that $\nu$ is a Lévy measure. We would like to point out that a similar equivalence of seminorms for absolutely continuous Lévy measures with completely monotone radial densities was obtained in [17].

In the remainder of this chapter we prove an analogous equivalence of norms for spaces $H^{\psi, \alpha}\left(\mathbb{R}^{n}\right)$, following the approach in [24] for Besov spaces.

Definition 2.7. For a function $f$ on $\mathbb{R}^{n}, h \in \mathbb{R}^{n}$ and $k \in \mathbb{N}$, the $k$ th difference of function $f$ is defined by $\left(\Delta_{h}^{k} f\right)(x):=\Delta_{h}^{1}\left(\Delta_{h}^{k-1} f\right)(x), x \in$ $\mathbb{R}^{n}$, where $\Delta_{h}^{1} f(x)=f(x+h)-f(x)$. Next, define the $k$-th modulus of continuity of a function $f \in L^{2}\left(\mathbb{R}^{n}\right)$ as $\omega_{k}(f, t)=\sup _{|h|<t}\left\|\Delta_{h}^{k} f\right\|_{L^{2}\left(\mathbb{R}^{n}\right)}, t>$ 0. Furthermore, for an admissible sequence $\left(\gamma_{j}\right)_{j \in \mathbb{N}_{0}}$ let $\bar{\gamma}_{j}=\sup _{k} \frac{\gamma_{j+k}}{\gamma_{k}}$ and $\underline{\gamma}_{j}=\inf _{k} \frac{\gamma_{j+k}}{\gamma_{k}}$, and let $\underline{s}(\gamma):=\lim _{j \rightarrow \infty} j^{-1} \log \underline{\gamma}_{j}$ and $\bar{s}(\gamma):=\lim _{j \rightarrow \infty} j^{-1} \log \bar{\gamma}_{j}$ be the lower and upper Boyd index, respectively.

Since $\gamma_{j+i+k} \leq \bar{\gamma}_{j} \gamma_{i+k}$ for all $i, j, k \in \mathbb{N}_{0}$ it follows that $\bar{\gamma}_{j+i} \leq \bar{\gamma}_{j} \bar{\gamma}_{i}$, so the sequence $\log \bar{\gamma}_{j}$ is subadditive. By Fekete's subadditive lemma the sequence $\left(\frac{\log \bar{\gamma}_{j}}{j}\right)_{j}$ converges to $\inf _{j} \frac{\log \bar{\gamma}_{j}}{j}$, so the upper index $\bar{s}(\gamma)$ is well defined. The analogous conclusion follows for the lower index $\underline{s}(\gamma)$, since $\log \underline{\gamma}_{j}=-\log \left(\overline{\gamma^{-1}}{ }_{j}\right)$.

TheOREM 2.8. [21, Theorem 4.1] Let $\sigma$ and $N$ be admissible sequences and $\underline{N}_{1}=\inf _{k \geq 0} \frac{N_{k+1}}{N_{k}}>1$ and $\frac{\underline{s}(\sigma)}{\bar{s}(N)}>0$. Let $k$ be an integer such that 
$k>\frac{\bar{s}(\sigma)}{\underline{s}(N)}$. Then the norm $\|\cdot\|_{B, \sigma, N}$ on $B_{2}^{\sigma, N}$ is equivalent to

$$
\|u\|_{L^{2}\left(\mathbb{R}^{n}\right)}+\left(\sum_{j=0}^{\infty} \sigma_{j}^{2} \omega_{k}\left(u, N_{j}^{-1}\right)^{2}\right)^{\frac{1}{2}} .
$$

For a similar result see also [20, Theorem 16]. Note that for every $a \in \mathcal{A}$ the sequence $N^{a, r}$ satisfies the assumption $\underline{N}_{1}^{a, r}>1$. Furthermore, recall that $\sigma^{\alpha}=\left(2^{\alpha j}\right)_{j \in \mathbb{N}_{0}}$ is an admissible sequence and by Remark 2.4 the sequence $N^{\psi, 2}$ is strongly increasing. One can easily show that $N^{\psi, 2}$ is also admissible. Furthermore,

$$
\frac{\underline{s}\left(\sigma^{\alpha}\right)}{\bar{s}\left(N^{\psi, 2}\right)} \geq \frac{\alpha}{2}>0 \text { and } \frac{\bar{s}\left(\sigma^{\alpha}\right)}{\underline{s}\left(N^{\psi, 2}\right)} \leq \frac{\alpha \log 2}{\frac{1}{\delta_{2}} \log 2}=\alpha \delta_{2},
$$

so Theorem 2.8 holds for $k>\alpha \delta_{2}$. Furthermore,

$$
\begin{aligned}
& \sum_{j=0}^{\infty} 2^{\alpha j} \sup _{|H|<1 / N_{j}^{\psi, 2}}\left\|\Delta_{H}^{k} u\right\|_{L^{2}\left(\mathbb{R}^{n}\right)}^{2} \\
& \quad \asymp \sum_{j=0}^{\infty} \int_{2^{-(j+1)} \leq t<2^{-j}} \frac{1}{t^{1+2 \alpha}} \sup _{|H|<1 / N_{j}^{\psi, 2}}\left\|\Delta_{H}^{k} u\right\|_{L^{2}\left(\mathbb{R}^{n}\right)}^{2} d t \\
& \quad \asymp \int_{0}^{1} \frac{1}{t^{1+2 \alpha}} \sup _{|H|<1 / \psi^{-1}\left(t^{-2}\right)}\left\|\Delta_{H}^{k} u\right\|_{L^{2}\left(\mathbb{R}^{n}\right)}^{2} d t
\end{aligned}
$$

since by $2^{-(j+1)} \leq t<2^{-j}$ implies $\psi^{-1}\left(t^{-2}\right) \asymp N_{j}^{\psi, 2}$. By change of variable $t^{-2}=\psi\left(|h|^{-1}\right)$ it follows that (2.4) is comparable to

$$
\int_{|h|<1} \frac{\left(\psi^{\alpha}\right)^{\prime}\left(|h|^{-1}\right)}{|h|^{n+1}} \sup _{|H|<|h|}\left\|\Delta_{H}^{k} u\right\|_{L^{2}\left(\mathbb{R}^{n}\right)}^{2} d h .
$$

Since $\left(\psi^{\alpha}\right)^{\prime}(t) \asymp \frac{\psi^{\alpha}(t)}{t}$ it follows that the last line is comparable to

$$
\int_{|h|<1} \frac{\psi^{\alpha}\left(|h|^{-1}\right)}{|h|^{n}} \sup _{|H|<|h|}\left\|\Delta_{H}^{k} u\right\|_{L^{2}\left(\mathbb{R}^{n}\right)}^{2} d h .
$$

REMARK 2.9. (i) By applying a straightforward generalization of [24, Theorem 2.6.1] to (2.5), Theorem 2.8, Theorem 2.5 and the calculation above imply that the norms

$$
\|u\|_{(1), \alpha, k}:=\|u\|_{L^{2}\left(\mathbb{R}^{n}\right)}+\left(\int_{h \mid<1} \frac{\psi^{\alpha}\left(|h|^{-1}\right)}{|h|^{n}}\left\|\Delta_{h}^{k} u\right\|_{L^{2}\left(\mathbb{R}^{n}\right)}^{2} d h\right)^{\frac{1}{2}}
$$

are equivalent to $\|\cdot\|_{\psi, \alpha}$, for all $k>\alpha \delta_{2}$. 
(ii) Since the function $\frac{\psi^{\alpha}\left(|\cdot|^{-1}\right)}{|\cdot|^{n}}$ is continuous and $\left\|\Delta_{h}^{k} u\right\|_{L^{2}} \leq c(k)\|u\|_{L^{2}}$ the norms $\|\cdot\|_{(1), \alpha, k}^{h_{0}}$,

$$
\|u\|_{(1), \alpha, k}^{h_{0}}:=\|u\|_{L^{2}\left(\mathbb{R}^{n}\right)}+\left(\int_{h \mid<h_{0}} \frac{\psi^{\alpha}\left(|h|^{-1}\right)}{|h|^{n}}\left\|\Delta_{h}^{k} u\right\|_{L^{2}\left(\mathbb{R}^{n}\right)}^{2} d h\right)^{\frac{1}{2}},
$$

are equivalent for all $h_{0}>0$.

(iii) Let $c>0, N \in \mathbb{Z}$ and $k>\alpha \delta_{2}$. The norm $\|\cdot\|_{(2)}^{c, N}$ on $H^{\psi, \alpha}\left(\mathbb{R}^{n}\right)$ defined by

$$
\|u\|_{(2), \alpha, k}^{c, N}=\|u\|_{L^{2}\left(\mathbb{R}^{n}\right)}+\left(\sum_{j=N}^{\infty} \psi^{\alpha}\left(2^{j}\right) 2^{n j} \int_{|h|<c 2^{-j}}\left\|\Delta_{h}^{k} u\right\|_{L^{2}\left(\mathbb{R}^{n}\right)}^{2} d h\right)^{\frac{1}{2}}
$$

is equivalent to the norm $\|\cdot\|_{(1)}$. This follows by applying (1.5) to the norm in (ii) for $h_{0}=c 2^{-N}$,

$$
\begin{aligned}
& \int_{|h|<c 2^{-N}} \frac{\psi^{\alpha}\left(|h|^{-1}\right)}{|h|^{n}}\left\|\Delta_{h}^{k} u\right\|_{L^{2}\left(\mathbb{R}^{n}\right)}^{2} d h \\
& =\sum_{j=N}^{\infty} \int_{c 2^{-j-1} \leq|h|<c 2^{-j}} \frac{\psi^{\alpha}\left(|h|^{-1}\right)}{|h|^{n}}\left\|\Delta_{h}^{k} u\right\|_{L^{2}\left(\mathbb{R}^{n}\right)}^{2} d h \\
& \asymp \sum_{j=N}^{\infty} \psi^{\alpha}\left(2^{j}\right) 2^{n j} \int_{c 2^{-j-1} \leq|h|<c 2^{-j}}\left\|\Delta_{h}^{k} u\right\|_{L^{2}\left(\mathbb{R}^{n}\right)}^{2} d h \\
& \asymp \sum_{j=N}^{\infty} \sum_{i=N}^{j} \psi^{\alpha}\left(2^{i}\right) 2^{n i} \int_{c 2^{-j-1} \leq|h|<c 2^{-j}}\left\|\Delta_{h}^{k} u\right\|_{L^{2}\left(\mathbb{R}^{n}\right)}^{2} d h \\
& =\sum_{i=N}^{\infty} \psi^{\alpha}\left(2^{i}\right) 2^{n i} \int_{|h|<c 2^{-j}}\left\|\Delta_{h}^{k} u\right\|_{L^{2}\left(\mathbb{R}^{n}\right)}^{2} d h .
\end{aligned}
$$

\section{THE RESTRICTION THEOREM}

In this section we provide a detailed proof of the continuity of the restriction operator, as a generalization of [18, Section V.1.2]. The same approach is used in [20, Appendix III]. Before we start with the proof, we show the following useful consequence of the estimates on Bessel-type potentials from Lemma 2.6. 
Lemma 3.1. Let $d \leq n, D$ a d-set in $\mathbb{R}^{n}$ and $\mu$ the $d$-measure on $D$. Let $\psi$ be a function such that (1.2), (H1) and (H2) hold and $\alpha>0$ such that

$$
\frac{n-d}{2}<\alpha \delta_{1} \leq \alpha\left(\delta_{2} \vee \delta_{4}\right)<\frac{n-d}{2}+1 \text {. }
$$

Then there exists a constant $c>0$ such that for all $r \leq \frac{1}{3}$ and $f \in L^{2}\left(\mathbb{R}^{n}\right)$

$$
\iint_{|x-y|<r}\left(K_{\psi, \alpha} * f(x)-K_{\psi, \alpha} * f(y)\right)^{2} \mu(d x) \mu(d y) \leq c \frac{r^{2 d-n}}{\phi^{\alpha}\left(r^{-2}\right)}\|f\|_{L^{2}\left(\mathbb{R}^{n}\right)}^{2} .
$$

Proof. Note that for every constant $0<a<1$

$$
\begin{aligned}
\left(K_{\psi, \alpha} * f(x)-K_{\psi, \alpha} * f(y)\right)^{2} \leq & \int\left|K_{\psi, \alpha}(x-t)-K_{\psi, \alpha}(y-t)\right|^{2 a} f^{2}(t) d t \\
& \cdot \int\left|K_{\psi, \alpha}(x-t)-K_{\psi, \alpha}(y-t)\right|^{2(1-a)} d t .
\end{aligned}
$$

Let $|x-y|<r$. By Lemma 2.6 it follows that

$$
\begin{aligned}
& \int_{|y-t|<2 r}\left|K_{\psi, \alpha}(x-t)-K_{\psi, \alpha}(y-t)\right|^{2(1-a)} d t \\
& \quad \lesssim \int_{|z|<3 r}\left|K_{\psi, \alpha}(z)\right|^{2(1-a)} d z \lesssim \int_{|z|<3 r}\left(\frac{1}{|z|^{n} \phi^{\frac{\alpha}{2}}\left(|z|^{-2}\right)}\right)^{2(1-a)} d z \\
& \quad \stackrel{(\mathrm{H} 1)}{\lesssim} \frac{r^{-2 \alpha \delta_{1}(1-a)}}{\left(\phi^{\frac{\alpha}{2}}\left(r^{-2}\right)\right)^{2(1-a)}} \int_{|z|<3 r}\left(\frac{1}{|z|^{n-\alpha \delta_{1}}}\right)^{2(1-a)} d z \\
& \quad=\widetilde{c}_{1} \frac{r^{n}}{\left(r^{n} \phi^{\frac{\alpha}{2}}\left(r^{-2}\right)\right)^{2(1-a)}},
\end{aligned}
$$

for some $\widetilde{c}_{1}>0$ and $a$ such that

$$
2(1-a)\left(n-\alpha \delta_{1}\right)<n .
$$

Analogously, if

$$
2 a\left(n-\alpha \delta_{1}\right)<d
$$

then for all $t \in \mathbb{R}^{n}$ there exists a constant $\widetilde{c}_{2}>0$

$$
\iint_{\substack{|x-y|<r \\|y-t|<2 r}}\left|K_{\psi, \alpha}(x-t)-K_{\psi, \alpha}(y-t)\right|^{2 a} \mu(d x) \mu(d y) \leq \widetilde{c}_{2} \frac{r^{2 d}}{\left(r^{n} \phi^{\frac{\alpha}{2}}\left(r^{-2}\right)\right)^{2 a}} .
$$

Therefore, it follows that

$$
\begin{aligned}
& \iint_{|x-y|<r}\left(\int_{|y-t|<2 r}\left(K_{\psi, \alpha}(x-t)-K_{\psi, \alpha}(y-t)\right) f(t) d t\right)^{2} \mu(d x) \mu(d y) \\
& \leq \widetilde{c}_{1} \widetilde{c}_{2} \frac{r^{2 d-n}}{\phi^{\alpha}\left(r^{-2}\right)}\|f\|_{L^{2}\left(\mathbb{R}^{n}\right)}^{2} .
\end{aligned}
$$


For the second part, note that the mean value theorem and Lemma 2.6 imply that

$$
\begin{aligned}
& \iint_{\substack{|x-y|<r \\
|y-t|>2 r}}\left|K_{\psi, \alpha}(x-t)-K_{\psi, \alpha}(y-t)\right|^{2 a} \mu(d x) \mu(d y) \\
& \lesssim r^{2 a} \iint_{\substack{|x-y|<r \\
|y-t|>2 r}}\left(\frac{1}{\left|z_{x, y}\right|^{n+1} \phi^{\frac{\alpha}{2}}\left(\left|z_{x, y}\right|^{-2}\right)}\right)^{2 a} \mu(d x) \mu(d y),
\end{aligned}
$$

where $z_{x, y}=y-t+\theta_{x, y}(x-y)$ for some $\theta_{x, y} \in(0,1)$ and $\left|z_{x, y}\right| \geq \frac{|y-t|}{2}$. Let $\delta=\delta_{2} \vee \delta_{4}$. By (H1) and (H2) there exists a constant $\widetilde{c}_{3}>0$ such that the last line in (3.5) is comparably less then

$$
\frac{r^{2 a+d}}{\left(r^{\alpha \delta} \phi^{\frac{\alpha}{2}}\left(r^{-2}\right)\right)^{2 a}} \int_{|z|>2 r}\left(\frac{1}{|z|^{n+1-\alpha \delta}}\right)^{2 a} \mu(d z)=\widetilde{c}_{3} \frac{r^{2 d}}{\left(r^{n} \phi^{\frac{\alpha}{2}}\left(r^{-2}\right)\right)^{2 a}}
$$

if

$$
2 a(n+1-\alpha \delta)>d \text {. }
$$

Similarly, if

$$
2(1-a)(n+1-\alpha \delta)>n
$$

then there exists a constant $\widetilde{c}_{4}>0$ such that $|x-y|<r$ implies

$$
\int_{2 r<|y-t|}\left|K_{\psi, \alpha}(y-t)-K_{\psi, \alpha}(x-t)\right|^{2(1-a)} d t \leq \widetilde{c}_{4} \frac{r^{n}}{\left(r^{n} \phi^{\frac{\alpha}{2}}\left(r^{-2}\right)\right)^{2(1-a)}} .
$$

Therefore, it follows that

$$
\begin{aligned}
& \iint_{|x-y|<r}\left(\int_{|y-t|>2 r}\left(K_{\psi, \alpha}(x-t)-K_{\psi, \alpha}(y-t)\right) f(t) d t\right)^{2} \mu(d x) \mu(d y) \\
& \leq \widetilde{c}_{3} \widetilde{c}_{4} \frac{r^{2 d-n}}{\phi^{\alpha}\left(r^{-2}\right)}\|f\|_{L^{2}\left(\mathbb{R}^{n}\right)}^{2} .
\end{aligned}
$$

Since $\frac{n-d}{2}<\alpha \delta_{1} \leq \alpha \delta<\frac{n-d}{2}+1$ it follows that

$$
\left(\frac{d}{2(n+1-\alpha \delta)}, 1-\frac{n}{2(n+1-\alpha \delta)}\right) \cap\left(1-\frac{n}{2\left(n-\alpha \delta_{1}\right)}, \frac{d}{2\left(n-\alpha \delta_{1}\right)}\right) \neq \emptyset,
$$

so we can choose $a$ such that (3.3), (3.4), (3.6) and (3.7) hold. 
For a $d$-set $D$ in $\mathbb{R}^{n}$ with $d$-measure $\mu$ and $\alpha>0$ such that (3.1) holds, we define the trace space in terms of differences

$$
\begin{aligned}
& H^{\psi, \alpha}(D, \mu)=\left\{u \in L^{2}(D, \mu):\|u\|_{(1), D, \mu}<\infty\right\} \\
& \|u\|_{(1), D, \mu}:=\|u\|_{L^{2}}+\left(\iint_{|x-y|<1}|u(x)-u(y)|^{2} \frac{\psi^{\alpha}\left(|x-y|^{-1}\right)}{|x-y|^{2 d-n}} \mu(d x) \mu(d y)\right)^{\frac{1}{2}} .
\end{aligned}
$$

Note that, similarly as in Remark 2.9(iii), one can easily show that the norms $\|\cdot\|_{(1), D, \mu}$ and $\|\cdot\|_{(2), D, \mu}^{c, N}$ are comparable, where

$\|u\|_{(2), D, \mu}^{c, N}:=\|u\|_{L^{2}(D, \mu)}+\left(\sum_{j=N}^{\infty} \frac{\psi^{\alpha}\left(2^{j}\right)}{2^{-(2 d-n) j}} \iint_{|x-y|<c 2^{-j}}|u(x)-u(y)|^{2} \mu(d x) \mu(d y)\right)^{\frac{1}{2}}$.

Next we define the restriction operator. Let $R u$ be the pointwise restriction on $D$ of the strictly defined function corresponding to $u \in H^{\psi, \alpha}\left(\mathbb{R}^{n}\right)$, i.e.

$$
R u(x)=\lim _{r \rightarrow 0} \frac{1}{\lambda(B(x, r))} \int_{B(x, r)} u(y) d y, x \in D,
$$

whenever the limit exists. Here, instead of $u$ one can a take a quasi continuous modification $\widetilde{u}$ of $u$, see for example [5, Theorem 3.5.7]. Recall that a function $\widetilde{u}$ is quasi continuous if for every $\varepsilon>0$ there exists an open set $G_{\varepsilon}$ such that $\widetilde{u}_{\mid G_{\varepsilon}^{c}}$ is continuous, $\operatorname{Cap}_{\psi, \alpha}\left(G_{\varepsilon}\right)<\varepsilon$ and $\widetilde{u}=u$ a.e. This means that outside of set $N=\cap_{\varepsilon>0} G_{\varepsilon}$ function $u$ can be strictly defined and therefore $\widetilde{R} u=u$ on $N^{c}$. To show that $\mu(N)=0$, note that by the definition of capacity, for every $\varepsilon>0$ there exists a function $v \in H^{\psi, \alpha}\left(\mathbb{R}^{n}\right)$ such that $v \geq 1$ on $N$ and $\|v\|_{a, 1}<\varepsilon$, so

$$
\mu(N) \leq\left(\int_{N}|v(x)|^{2} \mu(d y)\right)^{\frac{1}{2}} \leq \widetilde{c}_{1}\|v\|_{\psi, \alpha}<\widetilde{c}_{1} \varepsilon
$$

and therefore $\mu(N)=0$. Here the second inequality follows from calculations analogous to ones in the proof of Lemma 3.1.

TheOrem 3.2. Let $d \leq n, D$ a d-set in $\mathbb{R}^{n}$ and $\mu$ the $d$-measure on $D$. Let $\psi$ be a function such that (1.2), (H1) and (H2) hold and $\alpha>0$ satisfying (3.1). There exists a continuous restriction operator $R: H^{\psi, \alpha}\left(\mathbb{R}^{n}\right) \rightarrow$ $H^{\psi, \alpha}(D, \mu)$.

Proof. Using the classical interpolation theorem for a special class of spaces associated with $H^{\psi, 1}\left(\mathbb{R}^{n}\right)$ we will show that there exists a constant $\widetilde{c}_{1}>0$ such that for all $u \in H^{\psi, \alpha}\left(\mathbb{R}^{n}\right)$

$$
\|R u\|_{(2), D, \mu}^{1,1} \leq \widetilde{c}_{1}\|u\|_{\psi, \alpha} .
$$


Denote by $a_{j}(x, y)=|R u(x)-R u(y)| 1_{\left\{|x-y|<2^{-j}\right\}}, j \in \mathbb{N}_{0}$ and let $T$ be an operator on $L^{2}\left(\mathbb{R}^{n}\right)$ such that $T u=\left(a_{j}\right)_{j \in \mathbb{N}_{0}}$. Since $R u=u \mu$-a.e. on $D$, by Lemma 3.1 there exists a constant $\widetilde{c}_{2}>0$ such that

$\sup _{j \in \mathbb{N}_{0}}\left(\phi^{\alpha}\left(2^{2 j}\right) 2^{j(2 d-n)} \iint_{|x-y|<2^{-j}}|R u(x)-R u(y)|^{2} \mu(d x) \mu(d y)\right) \leq \widetilde{c}_{2}\|f\|_{L^{2}\left(\mathbb{R}^{n}\right)}^{2}$ for all $u=K_{\psi, \alpha} * f, f \in L^{2}\left(\mathbb{R}^{n}\right)$. Let $L=L^{2}(D \times D, \mu \times \mu)$. It follows that

$$
\left(a_{j}\right)_{j} \in l_{\infty}^{\phi, \alpha}(L)=\left\{\left(\xi_{j}\right)_{j}: \xi_{j} \in L,\|\xi\|_{l_{\infty}^{\phi, \alpha}(L)}=\sup _{j \in \mathbb{N}_{0}} \phi^{\alpha}\left(2^{2 j}\right) 2^{n j}\left\|\xi_{j}\right\|_{L}^{2}<\infty\right\}
$$

and that the operator $T$ is bounded from $H^{\psi, \alpha}\left(\mathbb{R}^{n}\right)$ to $l_{\infty}^{\phi, \alpha}(L)$, i.e.

$$
\left\|\left(a_{j}\right)_{j \in \mathbb{N}_{0}}\right\|_{l_{\infty}^{\phi, \alpha}(L)} \leq \widetilde{c}_{2}\left\|K_{\psi, \alpha} * f\right\|_{\psi, \alpha}=\widetilde{c}_{2}\|f\|_{L^{2}\left(\mathbb{R}^{n}\right)} .
$$

Let $\left(X_{1}, X_{2}\right)_{\theta, 2}:=\left\{a \in X_{1}+X_{2}:\|a\|_{\left(X_{1}, X_{2}\right)_{\theta, 2}}<\infty\right\}$ be the $K$-interpolation space of Banach spaces $X_{1}$ and $X_{2}$ and $\|a\|_{\left(X_{1}, X_{2}\right)_{\theta, 2}}=\left(\int_{0}^{\infty}\left(t^{-\theta} K(t, a)\right)^{2} \frac{d t}{t}\right)^{\frac{1}{2}}$, where the Peetre $K$-functional is defined by

$$
K(t, a)=\inf _{a=a_{1}+a_{2}}\left(\left\|a_{1}\right\|_{X_{1}}+t\left\|a_{2}\right\|_{X_{2}}\right),
$$

see [25, Section 1.3, p.23]. Choose $\alpha_{0}<\alpha<\alpha_{1}$ such that

$$
\frac{n-d}{2}<\delta_{1} \alpha_{0} \leq\left(\delta_{2} \vee \delta_{4}\right) \alpha_{1}<\frac{n-d}{2}+1
$$

and let $\theta=\frac{\alpha_{1}-\alpha}{\alpha_{1}-\alpha_{0}} \in(0,1)$. By [25, Theorem 1.3.3(a)] and [2, Lemma 4.1], since $T$ is bounded from $H^{\psi, \alpha_{i}}\left(\mathbb{R}^{n}\right)$ to $l_{\infty}^{\phi, \alpha_{i}}(L), i=0,1$, it is also bounded from $\left(H^{\psi, \alpha_{0}}\left(\mathbb{R}^{n}\right), H^{\psi, \alpha_{1}}\left(\mathbb{R}^{n}\right)\right)_{\theta, 2}$ to $\left(l_{\infty}^{\phi, \alpha_{0}}(L), l_{\infty}^{\phi, \alpha_{1}}(L)\right)_{\theta, 2}$. By a version of $[25$, Theorem 1.18.2], $\left(l_{\infty}^{\phi, \alpha_{0}}(L), l_{\infty}^{\phi, \alpha_{1}}(L)\right)_{\theta, 2}=l_{2}^{\phi, \alpha}(L)$, where

$$
l_{2}^{\phi, \alpha}(L)=\left\{\left(\xi_{j}\right)_{j \in \mathbb{N}_{0}}: \xi_{j} \in L,\|\xi\|_{l_{2}^{\phi}(L)}=\left(\sum_{j \in \mathbb{N}_{0}} \phi^{\alpha}\left(2^{2 j}\right) 2^{n j}\left\|\xi_{j}\right\|_{L}^{2}\right)^{\frac{1}{2}}<\infty\right\} .
$$

Furthermore, $\left(l_{2}^{\phi, \alpha_{0}}\left(L^{2}\left(\mathbb{R}^{n}\right)\right), l_{2}^{\phi, \alpha_{1}}\left(L^{2}\left(\mathbb{R}^{n}\right)\right)\right)_{\theta, 2}=l_{2}^{\phi, \alpha}\left(L^{2}\left(\mathbb{R}^{n}\right)\right)$ and $H^{\psi, \alpha}\left(\mathbb{R}^{n}\right)$ is a retract of the space $l_{2}^{\phi, \alpha}\left(L^{2}\left(\mathbb{R}^{n}\right)\right)$, [4, Theorem 2.5 and Theorem 3.4]. Therefore, the interpolation identity $\left(H^{\psi, \alpha_{0}}\left(\mathbb{R}^{n}\right), H^{\psi, \alpha_{1}}\left(\mathbb{R}^{n}\right)\right)_{\theta, 2}=H^{\psi, \alpha}\left(\mathbb{R}^{n}\right)$ follows by $[4$, Theorem 5.3]. Combining these results we get (3.8).

\section{EXTENSION THEOREM}

The proof of the extension theorem is divided into two parts: case $d<n$ and case $d=n$. In the first case we define the extension operator $E$ by (4.3), using the Whitney decomposition of $D^{c}$ and the approach as in [18, V.1.3], which deals with the classical Besov spaces. The assumption $d<n$ is crucial in order to prove boundedness of the operator $E: L^{2}(D, \mu) \rightarrow L^{2}\left(\mathbb{R}^{n}\right)$, see 
the calculation prior to (4.6). The case $d=n$ follows then by interpreting the $n$-set $D$ in $\mathbb{R}^{n}$ as a $n$-subset of $\mathbb{R}^{n+1}$. The extension operator is then defined as a composition of the extension operator to $\mathbb{R}^{n+1}$ and restriction operator to $\mathbb{R}^{n}$.

Before proving the extension theorem for $d<n$, we recall the definition of the Whitney decomposition of a set.

Definition 4.1. A Whitney decomposition of an open set $A$ is a collection of closed cubes $\left\{Q_{i}\right\}_{i \in \mathbb{N}}$ with disjoint interiors and sides parallel to the axes such that $A=\bigcup_{i} Q_{i}$, where each cube $Q_{i}$ has side length $s_{i}=2^{-M_{i}}$ for some $M_{i} \in \mathbb{Z}$ and diameter $l_{i}$ such that $l_{i} \leq d\left(Q_{i}, A^{c}\right) \leq 4 l_{i}$.

Denote by $x_{i}$ the center of the cube $Q_{i}$ and let $\varepsilon \in\left(0, \frac{1}{4}\right)$. Denote by $Q_{i}^{*}=(1+\varepsilon) Q_{i}$ the cube with center $x_{i}$ expanded by factor $1+\varepsilon$. If $x \in Q_{k} \cap Q_{i}^{*}$ then

$$
\frac{1}{4} s_{k} \leq s_{i} \leq 4 s_{k}
$$

and $Q_{i}$ and $Q_{k}$ touch each other. This implies that every point in $A$ is covered by at most $N_{0}$ cubes $Q_{i}^{*}$, where $N_{0} \in \mathbb{N}$ depends only on $n$. By [18, Section I.2.3] we can associate with decomposition $\left\{Q_{i}^{*}\right\}$ a partition of unity $\left\{\varphi_{i}\right\}_{i \in \mathbb{N}} \subset C_{c}^{\infty}\left(\mathbb{R}^{n}\right)$, i.e. a family of non-negative functions with the following properties:

(4.2) $\operatorname{supp} \varphi_{i} \subset Q_{i}^{*}, \quad \sum \varphi_{i}=1$ on $D^{c}, \quad\left|D^{j} \varphi_{i}\right| \leq \widetilde{c}_{i}^{-|j|}$ for some $\widetilde{c}>0$.

If $D$ is a $d$-set in $\mathbb{R}^{n}$ then by [18, Proposition VIII.1.1] the closure $\bar{D}$ of $D$ is also a $d$-set and $\mu(\bar{D} \backslash D)=0$ for every $d$-measure $\mu$. Therefore, it is enough to prove the theorem for a closed $d$-set $D$.

Next, we define the extension operator $E$ from $H^{\psi, \alpha}(D, \mu)$ to $H^{\psi, \alpha}\left(\mathbb{R}^{n}\right)$, when $d<n$. Let $\omega_{i}=\mu\left(B\left(x_{i}, 6 l_{i}\right)\right)^{-1} \asymp l_{i}^{-d}$ and $I=\left\{i \in \mathbb{N}: s_{i} \leq 1\right\}$. For $H^{\psi, \alpha}(D, \mu)$ define

$$
E u(x)= \begin{cases}u(x), & x \in D, \\ \sum_{i \in I} \varphi_{i}(x) \omega_{i} \int_{\left|y-x_{i}\right|<6 l_{i}} u(y) \mu(d y), & x \notin D .\end{cases}
$$

THEOREM 4.2. Let $D$ be a closed $d$-set in $\mathbb{R}^{n}$ and $d<n$. Let $\psi$ be a function such that (1.2), (H1) and (H2) hold and $\alpha>0$ such that (3.1) holds. There exists a continuous extension operator $E: H^{\psi, \alpha}(D, \mu) \rightarrow H^{\psi, \alpha}\left(\mathbb{R}^{n}\right)$, given by (4.3), such that $E u=u \mu$-a.e. on $D$ for all $u \in H^{\psi, \alpha}(D, \mu)$.

Proof. We will show that for some $c>0, N \in \mathbb{N}$ and $k_{0}>\alpha \delta_{2}$ there exists a constant $\widetilde{c}_{1}$ such that

$$
\|E u\|_{(1), \alpha, k_{0}}^{h_{0}} \leq \widetilde{c}_{1}\|u\|_{(2), D, \mu}^{c, N}, \quad \forall u \in H^{\psi, \alpha}(D, \mu) .
$$


Here we can choose the smallest $k_{0} \in \mathbb{N}$ satisfying the given condition, see Theorem 2.8 and Remark 2.9. Since $D$ is of Lebesgue measure zero in $\mathbb{R}^{n}$ it is enough to prove (4.4) for $E u 1_{D^{c}}$. For every $x \in D^{c}$ there exists a $k$ such that $x \in Q_{k}$. If $s_{k}>4$ then by (4.2) $x \notin Q_{i}^{*}$ for all $i \in I$ and $E u(x)=0$. Therefore it is enough to consider the case when $s_{k} \leq 4$. Also note that $\sum_{i} \varphi_{i}(x)=\sum_{i \in I} \varphi_{i}(x)$ when $s_{k}<1 / 4$.

Let $x \in Q_{k}$ and let $i \in I$ be such that $\phi_{i}(x) \neq 0$. Then for all $y \in B\left(x_{i}, 6 l_{i}\right)$ we have

$$
\left|y-x_{k}\right| \leq\left|y-x_{i}\right|+\left|x_{i}-x\right|+\left|x-x_{k}\right| \leq 6 l_{i}+l_{i}+l_{k} \leq 29 l_{k},
$$

which implies that

$$
\begin{aligned}
|E u(x)| & \leq \sum_{i \in I} \varphi_{i}(x) \omega_{i} \int_{\left|y-x_{i}\right|<6 l_{i}}|u(y)| \mu(d y) \\
& \lesssim \sum_{i \in I} \varphi_{i}(x) l_{i}^{-d} \int_{\left|y-x_{k}\right|<29 l_{k}}|u(y)| \mu(d y) \\
& \stackrel{(4.1)}{\lesssim} \sum_{i \in I} \varphi_{i}(x) \cdot l_{k}^{-d} \int_{\left|y-x_{k}\right|<29 l_{k}}|u(y)| \mu(d y) \\
& \lesssim\left(l_{k}^{-d} \int_{\left|y-x_{k}\right|<29 l_{k}} u^{2}(y) \mu(d y)\right)^{1 / 2} .
\end{aligned}
$$

For $j \in \mathbb{N}$ define $\Delta_{j}:=\bigcup_{\left\{k: s_{k}=2^{-j}\right\}} Q_{k}$. Note that there exists an integer $N_{1}$ depending only on $n$ such that every point $y \in \mathbb{R}^{n}$ is covered by at most $N_{1}$ balls $B\left(x_{k}, 29 l_{k}\right)$ where $Q_{k} \subset \Delta_{j}$. This follows from the fact that $\left|x_{k}-x_{k^{\prime}}\right| \geq$ $2^{-j}$ and $l_{k}=\sqrt{n} 2^{-j}$, for all $Q_{k}, Q_{k^{\prime}} \subset \Delta_{j}$. By the previous calculation it follows that

$$
\begin{aligned}
\int_{D^{c}}|E u(x)|^{2} d x & =\sum_{j=-2}^{\infty} \sum_{Q_{k} \subset \Delta_{j}} \int_{Q_{k}}|E u(x)|^{2} d x \\
& \lesssim \sum_{j=-2}^{\infty} \sum_{Q_{k} \subset \Delta_{j}} \int_{Q_{k}}\left(l_{k}^{-d} \int_{\left|y-x_{k}\right|<29 l_{k}} u^{2}(y) \mu(d y)\right) d x \\
& \asymp \sum_{j=-2}^{\infty} 2^{-(n-d) j} \sum_{Q_{k} \subset \Delta_{j}} \int_{\left|y-x_{k}\right|<29 l_{k}} u^{2}(y) \mu(d y) \\
& \leq 2^{2(n-d)} \sum_{j=-2}^{\infty} \int_{\Delta_{j}} u^{2}(y) \mu(d y),
\end{aligned}
$$

which implies that

$$
\|E u\|_{L^{2}\left(\mathbb{R}^{n}\right)} \lesssim\|u\|_{L^{2}(D, \mu)} .
$$


Next, for $x \in \Delta_{i}, y \in \Delta_{j}$ and $|x-y|<2^{-i} / 2$ we have

$$
2^{-j} \sqrt{n} \leq d\left(\Delta_{j}, D\right) \leq d(y, D) \leq|x-y|+d(x, D) \leq 6 \sqrt{n} 2^{-i},
$$

so $j \geq i-2$. Analogously, $\sqrt{n} 2^{-i} \leq 5 \sqrt{n} 2^{-j}+2^{-i-1}$ so $j \leq i+2$. Therefore,

$$
x \in \Delta_{i},|x-y|<2^{-i} / 2 \Rightarrow y \in \bigcup_{j=i-2}^{i+2} \Delta_{j} .
$$

Since $E u(x)=0$ for for $x \in \Delta_{i}, i \leq-3$ it follows that $E u(y)=0$ when $|x-y|<2^{-i-1}$ for some $x \in \Delta_{i}, i \leq-5$. Analogously, $\Delta_{h}^{k_{0}}(E u)(x)=0$ if $|h|<2^{-5} / k_{0}$. Therefore, for $h_{i}:=2^{-i-1} / k_{0}$,

$$
\begin{aligned}
& \int_{D^{c}} \int_{|h|<h_{0}}\left|\Delta_{h}^{k_{0}}(E u)(x)\right|^{2} \frac{\psi^{\alpha}\left(|h|^{-1}\right)}{|h|^{n}} d h d x \\
& \leq \sum_{i=-4_{x \in \Delta_{i}}}^{\infty} \iint_{\substack{x\left|<h_{i}\\
\right| h \mid}}\left|\Delta_{h}^{k_{0}}(E u)(x)\right|^{2} \frac{\psi^{\alpha}\left(|h|^{-1}\right)}{|h|^{n}} d h d x \\
& +\sum_{i=5}^{\infty} \iint_{\substack{x \in \Delta_{i} \\
h_{i} \leq|h|<2^{-5} / k_{0}}}\left|\Delta_{h}^{k_{0}}(E u)(x)\right|^{2} \frac{\psi^{\alpha}\left(|h|^{-1}\right)}{|h|^{n}} d h d x=: A+B .
\end{aligned}
$$

First we asses the term $B$. Let $F_{i}:=\bigcup_{j=i}^{\infty} \Delta_{j}$. Note that

$$
\begin{aligned}
B & =\sum_{i=5}^{\infty} \sum_{m=4}^{i-1} \int_{h_{m+1} \leq|h|<h_{m}} \frac{\psi^{\alpha}\left(|h|^{-1}\right)}{|h|^{n}} \int_{x \in \Delta_{i}}\left|\Delta_{h}^{k_{0}}(E u)(x)\right|^{2} d x d h \\
& =\sum_{m=h_{h_{m+1}} \leq|h|<h_{m}}^{\infty} \frac{\psi^{\alpha}\left(|h|^{-1}\right)}{|h|^{n}} \int_{x \in F_{m+1}}\left|\Delta_{h}^{k_{0}}(E u)(x)\right|^{2} d x d h \\
& \stackrel{(1.5)}{\lesssim} \sum_{m=4}^{\infty} \psi^{\alpha}\left(2^{m}\right) 2^{m n} \int_{\substack{x \in F_{m+1} \\
h_{m+1} \leq|h|<h_{m}}}\left|\Delta_{h}^{k_{0}}(E u)(x)\right|^{2} d x d h .
\end{aligned}
$$

Similarly as in (4.7), for $x \in F_{i+1}$ and $|h|<h_{i}$ it follows that $x+\ell h \in F_{i-2}$, for all $\ell \in\left\{0,1, \ldots k_{0}\right\}$. Since

$\left|\Delta_{h}^{k_{0}}(E u)(x)\right|^{2} \lesssim|E u(x)-E u(x+h)|^{2}+\cdots+\left|E u\left(x+\left(k_{0}-1\right) h\right)-E u\left(x+k_{0} h\right)\right|^{2}$ it follows that

$$
B \lesssim \sum_{m=4}^{\infty} \psi^{\alpha}\left(2^{m}\right) 2^{m n} \int_{\substack{x, y \in F_{m-2} \\|x-y|<h_{m}}}(E u(x)-E u(y))^{2} d x d y
$$


For $k, m \geq 2$ and $x \in \Delta_{k}$ and $y \in \Delta_{m}$ it follows that

$$
|E u(x)-E u(y)| \leq \sum_{i} \sum_{j} \varphi_{i}(x) \varphi_{j}(y) \omega_{i} \omega_{j} \iint_{\substack{\left|s-x_{i}\right|<6 l_{i} \\\left|t-x_{j}\right|<6 l_{j}}}|u(s)-u(t)| \mu(d s) \mu(d t) .
$$

From

$$
x \in \Delta_{k}, \varphi_{i}(x) \neq 0 \Rightarrow \frac{1}{8} l_{k} \leq l_{i} \leq 64 l_{k}
$$

it follows that

$$
\begin{aligned}
|E u(x)-E u(y)| & \stackrel{(4.5)}{\lesssim} \sum_{i} \sum_{j} \varphi_{i}(x) \varphi_{j}(y) l_{k}^{-d} l_{m}^{-d} \iint_{\begin{array}{c}
\left|s-x_{k}\right|<29 l_{k} \\
\left|t-x_{m}\right|<29 l_{m}
\end{array}}|u(s)-u(t)| \mu(d s) \mu(d t) \\
& \leq\left(\begin{array}{c}
l_{k}^{-d} l_{m}^{-d} \iint_{\substack{\left|s-x_{k}\right|<29 l_{k} \\
\left|t-x_{m}\right|<29 l_{m}}}(u(t)-u(s))^{2} \mu(d t) \mu(d s)
\end{array}\right)^{1 / 2} .
\end{aligned}
$$

Here $x_{k}, x_{m}$ are the centres and $l_{k}, l_{m}$ diameters of cubes $Q_{p} \subset \Delta_{k}$ and $Q_{r} \subset \Delta_{m}$ containing $x$ and $y$ respectively. Now it follows that for $i \in \mathbb{N}$, $y \in \Delta_{m}$ and $k, m \geq 2$

$$
\begin{aligned}
& \int_{\substack{x \in \Delta_{k},|x-y|<2^{-i}}}|E u(x)-E u(y)|^{2} d x \\
& \lesssim \int_{\substack{x \in \Delta_{k} \\
|x-y|<2^{-i}}} l_{k}^{-d} l_{m}^{-d} \iint_{\substack{\left|s-x_{k}\right|<29 l_{k} \\
\left|t-x_{m}\right|<29 l_{m}}}(u(t)-u(s))^{2} \mu(d t) \mu(d s) d x \\
& \leq N_{0} l_{k}^{-d} l_{m}^{-d} s_{k}^{-n} \underset{\substack{|s-y|<c 2^{-k}+2^{-i} \\
\left|t-x_{m}\right|<29 l_{m}}}{\int}(u(t)-u(s))^{2} \mu(d t) \mu(d s),
\end{aligned}
$$

where $c=30 \sqrt{n}$. Analogously, we get

$$
\begin{aligned}
& \iint_{\substack{x \in \Delta_{k}, y \in \Delta_{m} \\
|x-y|<2^{-i}}}|E u(x)-E u(y)|^{2} d x d y \\
& \lesssim N_{0}^{2} 2^{-(n-d) k} 2^{-(n-d) m} \iint_{|t-s|<2^{-i}+c 2^{-k}+c 2^{-m}}(u(t)-u(s))^{2} d \mu(s) d \mu(t) .
\end{aligned}
$$


This implies that for $i \geq 4$

$$
\begin{aligned}
& \iint_{\substack{x, y \in F_{i-2} \\
|x-y|<2^{-i}}}|E u(x)-E u(y)|^{2} d x d y=\sum_{\substack{k, m=i-2 \\
x \in \Delta_{k}, y \in \Delta_{m} \\
|x-y|<2^{-i}}}^{\infty}|E u(x)-E u(y)|^{2} d x d y \\
& \lesssim\left(\sum_{k, m=i-2}^{\infty} 2^{(d-n) k} 2^{(d-n) m}\right)_{|t-s|<(8 c+1) 2^{-i}}(u(t)-u(s))^{2} \mu(d s) \mu(d t)
\end{aligned}
$$

and by applying this to (4.8) we get that

$$
B \lesssim \sum_{i=4}^{\infty} \psi^{\alpha}\left(2^{i}\right) 2^{i(2 d-n)} \int_{|t-s|<(8 c+1) 2^{-i}}(u(t)-u(s))^{2} \mu(d s) \mu(d t) .
$$

Next, let

$$
F(h):=\sum_{|j|=k_{0}} \int_{\Delta_{i}} \int_{0}^{1} \ldots \int_{0}^{1}|h|^{2 k_{0}}\left|D^{j}(E u)\left(x+\left(t_{1}+\ldots+t_{k_{0}}\right) h\right)\right|^{2} d t_{1} \ldots d t_{k_{0}} d x .
$$

By the mean value theorem

$$
\begin{aligned}
A & \lesssim \sum_{i=-4}^{\infty} \int_{|h|<h_{i}} F(h) \frac{\psi^{\alpha}\left(|h|^{-1}\right)}{|h|^{n}} d h \\
& \stackrel{(4.7)}{\leq} \sum_{i=-4}^{\infty} \int_{|h|<h_{i}} \frac{\psi^{\alpha}\left(|h|^{-1}\right)}{|h|^{n-k_{0}}} d h \cdot \sum_{|j|=k_{0} F_{i-2} \backslash F_{i+3}} \int_{\left.D^{j}(E u)(z)\right|^{2} d z} \\
& \stackrel{(H 1)}{\lesssim} 5 \sum_{i=-2}^{\infty} \frac{\psi^{\alpha}\left(2^{i}\right)}{2^{2 k_{0} i}} \sum_{|j|=k_{0}} \int_{\Delta_{i}}\left|D^{j}(E u)(z)\right|^{2} d z .
\end{aligned}
$$

In the last line we also used that $D^{j}(E u)(z)=0$ if $z \in \Delta_{i}$ and $i \leq-3$. To find an upper bound for $\left|D^{j}(E u)(z)\right|, z \in \Delta_{i}$, we distinguish two cases; $i \geq 2$ and $i<2$. First, take $z, y \in Q_{m} \subset \Delta_{l}, l \geq 2$ and $|j|=k_{0}$. Since $\sum_{i} D^{j} \varphi_{i}(z)=0$, by similar calculations as before, we get

$$
\begin{aligned}
& \left|D^{j}(E u)(z)\right|=\left|\sum_{i} D^{j} \varphi_{i}(z) \omega_{i} \int_{\left|s-x_{i}\right|<6 l_{i}}(u(s)-E u(y)) \mu(d s)\right| \\
& \leq \sum_{i} \sum_{k}\left|D^{j} \varphi_{i}(z)\right| \varphi_{k}(y)\left(\omega_{i} \omega_{k} \iint_{\substack{\left|s-x_{i}\right|<6 l_{i} \\
\left|t-x_{k}\right|<6 l_{k}}}|u(s)-u(t)|^{2} \mu(d s) \mu(d t)\right)^{\frac{1}{2}} .
\end{aligned}
$$


Recall that there are at most $N_{0}$ indices $i$ for which $z \in Q_{i}^{*}$ and $D^{j} \varphi_{i}(z) \neq 0$. By (4.1) and (4.2), $z \in Q_{i}^{*}$ implies $\omega_{i} \asymp l_{m}^{-d}$ and $\left|D^{j} \varphi_{i}(z)\right| \lesssim l_{i}^{-|j|} \lesssim l_{m}^{-|j|}$. Also, by $(4.9) \omega_{k} \asymp l_{m}^{-d}$ for $k$ such that $\varphi_{k}(y) \neq 0$. Therefore,

$$
\left|D^{j}(E u)(z)\right| \stackrel{(4.5)}{\lesssim} l_{m}^{-k_{0}}\left(l_{m}^{-2 d} \iint_{\substack{\left|s-x_{m}\right|<29 l_{m} \\\left|t-x_{m}\right|<29 l_{m}}}|u(s)-u(t)|^{2} \mu(d s) \mu(d t)\right)^{\frac{1}{2}} .
$$

Applying this inequality to (4.11) we arrive to

$A \lesssim \sum_{i=2}^{\infty} \frac{\psi^{\alpha}\left(2^{i}\right)}{2^{2 k_{0} i}} \sum_{|j|=k_{0}} \sum_{Q_{m} \subset \Delta_{i}} \int_{Q_{m}} 2^{2 k_{0} i+2 i d} \iint_{\substack{\left|s-x_{m}\right|<29 l_{m} \\\left|t-x_{m}\right|<29 l_{m}}}|u(s)-u(t)|^{2} \mu(d s) \mu(d t) d z$ and since every $s \in D^{c}$ is covered by at most $N_{1}$ balls $B\left(x_{m}, 29 l_{m}\right)$ it follows that

$$
A \lesssim \sum_{i=2}^{\infty} \psi^{\alpha}\left(2^{i}\right) 2^{i(2 d-n)} \iint_{|s-t|<60 \sqrt{n} 2^{-i}}|u(s)-u(t)|^{2} \mu(d s) \mu(d t) .
$$

For the remaining part in $A$, take $z \in \Delta_{k}, k \geq-2$. By the same arguments as before,

$$
\begin{aligned}
\left|D^{j}(E u)(z)\right| & \leq \sum_{i}\left|D^{j} \varphi_{i}(z)\right| \omega_{i} \int_{\left|s-x_{i}\right|<6 l_{i}}|u(s)| \mu(d s) \\
& \stackrel{(4.2)}{\lesssim} \sum_{\varphi_{i}(z) \neq 0} l_{i}^{-2}\left(\omega_{i} \int_{\left|s-x_{i}\right|<6 l_{i}}|u(s)|^{2} \mu(d s)\right)^{\frac{1}{2}} \\
& \lesssim 2^{k k_{0}}\left(2^{k d} \int_{\left|s-x_{k}\right|<29 l_{k}}|u(s)|^{2} \mu(d s)\right)^{\frac{1}{2}}
\end{aligned}
$$

and therefore $\sum_{i=-2}^{1} \int_{\Delta_{i}}|D(E u)(z)|^{2} d z \lesssim\|u\|_{L^{2}(D, \mu)}$. This inequality together with (4.6), (4.10) and (4.12) implies (4.4).

That $E$ is truly the extension operator for $R$, i.e. that $R E u=u \mu$-a.e., follows from calculation similar to the calculation above. One first shows that 
for every $t_{0} \in D$ and $r>0$ small enough

$$
\begin{aligned}
& \int_{\left|x-t_{0}\right| \leq r}\left(E u(x)-u\left(t_{0}\right)\right)^{2} d x \\
& \quad \lesssim \frac{r^{d}}{\psi^{\alpha}\left(r^{-1}\right)} \int_{\left|t-t_{0}\right|<30 r}\left(u(t)-u\left(t_{0}\right)\right)^{2} \frac{\psi^{\alpha}\left(\left|t-t_{0}\right|^{-1}\right)}{\left|t-t_{0}\right|^{2 d-n}} \mu(d t),
\end{aligned}
$$

where the integral is finite for $\mu$-almost all $t_{0}$ and decreasing as $r$ goes to 0 . Since $\lim _{r \rightarrow 0} \frac{1}{\psi^{\alpha}\left(r^{-1}\right) r^{n-d}}=0$, it follows that for $\mu$ almost all $t_{0}$

$$
\left|R E u\left(t_{0}\right)-u\left(t_{0}\right)\right| \lesssim \lim _{r \rightarrow 0}\left(r^{-n} \int_{\left|x-t_{0}\right| \leq r}\left(E u(x)-u\left(t_{0}\right)\right)^{2} d x\right)^{1 / 2}=0
$$

Remark 4.3. Let $\alpha \in\left(\frac{1}{2}, \infty\right)$. Note that for a $n$-set $D$ in $\mathbb{R}^{n}, \widetilde{D}=D \times\{0\}$ is a $n$-set in $\mathbb{R}^{n+1}$ and that every function $u \in H^{\psi, \alpha}(D, \mu)$ can be represented as a function $\widetilde{u}$ in $H^{\widetilde{\psi}, \widetilde{\alpha}}(\widetilde{D}, \widetilde{\mu})$, where

$$
\begin{aligned}
& \widetilde{\alpha}:=2 \alpha, \widetilde{\psi}(|\xi|):=\psi^{\frac{1}{2}}(|\xi|)|\xi|^{\frac{1}{\alpha}}, \widetilde{\mu}(A \times\{0\}):=\mu(A) \\
& \widetilde{u}(x, 0):=u(x), x \in D \text { and }\|\widetilde{u}\|_{(1), \widetilde{D}, \widetilde{\mu}}=\|u\|_{(1), D, \mu} .
\end{aligned}
$$

By [22, Theorem 7.13.] the function $\xi \mapsto \phi^{\frac{1}{2}}(|\xi|)|\xi|^{\frac{1}{2 \alpha}}$ is a complete Bernstein function and $\widetilde{\psi}$ satisfies conditions (H1) and (H2) with $\widetilde{\delta}_{i}:=\frac{\delta_{i}}{2}+\frac{1}{4 \alpha}$. Analogously, the space $H^{\psi, \alpha}\left(\mathbb{R}^{n}\right)$ can be represented as $H^{\widetilde{\psi}, \widetilde{\alpha}}\left(\mathbb{R}^{n} \times\{0\}, \bar{\mu}\right)$, where $\bar{\mu}$ is the restriction of the $n$-dimensional Hausdorff measure in $\mathbb{R}^{n+1}$ to $\mathbb{R}^{n} \times\{0\}$.

TheOREM 4.4. Theorem 4.2 holds true as well in the case of $d=n$ and $\alpha \in\left(\frac{1}{2}, \infty\right)$.

Proof. Take $u \in H^{\psi, \alpha}(D, \mu)$ and let $\widetilde{u}$ be the corresponding function in $H^{\widetilde{\psi}, \widetilde{\alpha}}(\widetilde{D}, \widetilde{\mu})$ from Remark 4.3. By Theorem 4.2 function $\widetilde{u}$ can be extended to a function $\widetilde{E} \widetilde{u} \in H^{\widetilde{\psi}, \widetilde{\alpha}}\left(\mathbb{R}^{n+1}\right)$, which can then be restricted to a function in $H^{\widetilde{\psi}, \widetilde{\alpha}}\left(\mathbb{R}^{n} \times\{0\}, \bar{\mu}\right)$ by applying the continuous restriction operator $\bar{R}$ from Theorem 3.2. Again using Remark 4.3, we can define the extension operator $E$ as

$$
(E u)(x)=(\bar{R} \widetilde{E} \widetilde{u})(x, 0), x \in \mathbb{R}^{n} .
$$

The continuity of $E$ follows from the continuity of the extension and restriction operators $\widetilde{E}$ and $\bar{R}$ and $R E u=u$ almost everywhere on $D$, where $R$ is the restriction operator from $H^{\psi, \alpha}\left(\mathbb{R}^{n}\right)$ to $H^{\psi, \alpha}(D, \mu)$.

Proof of Corollary 1.3: By applying Theorem 1.2, the proof is an immediate consequence of [26, Theorem 1.1, Corollary 2.9]. By [26, Corollary 
2.9], $H_{0}^{\psi, 1}(D)=H^{\psi, 1}(D)$ if and only if the capacity of the boundary $\partial D$ with respect to the Lévy process with the characteristic exponent $\psi$ is equal 0, i.e.

$$
\operatorname{Cap}_{\psi}(\partial D) \asymp \inf \left\{\|u\|_{\psi, 1}: u \geq 1 \text { on } U, U \text { open and } \partial D \subset U\right\}=0 .
$$

Using the scaling condition (H1) we can relate this capacity to the Riesz capacities of order $n-2 \delta_{i}, i=1,2$,

$$
\operatorname{Cap}_{n-2 \delta_{1}}(\partial D) \leq \operatorname{Cap}_{\psi}(\partial D) \leq \operatorname{Cap}_{n-2 \delta_{2}}(\partial D) .
$$

By using the well known connection of the Riesz capacity and the Hausdorff dimension of a set (see e.g. [1]) wee conclude, similarly as in [26, Theorem 1.1], that conditions on $\delta_{2}$ in $(i)$ imply that $\operatorname{Cap}_{\psi}(\partial D)=0$. Analogously, conditions in $(i i)$ imply that $\operatorname{Cap}_{\psi}(\partial D)=0$.

\section{ACKNOWLEDGEMENTS.}

This work was supported by the Croatian Science Foundation under the project 3526 .

\section{REFERENCES}

[1] D. R. Adams and L. I. Hedberg, Function spaces and potential theory, Springer-Verlag, Berlin, 1996.

[2] A. Almeida and A. Caetano, Real interpolation of generalized Besov-Hardy spaces and applications J. Fourier Anal. Appl. 17 (2010), 691-719.

[3] K. Bogdan, K. Burdzy and Z.-Q. Chen, Censored stable processes, Probab. Theory Related Fields 127 (2003), 89-152.

[4] F. Cobos and D.L. Fernandez, Hardy-Sobolev spaces and Besov spaces with a function parameter, in Function Spaces and Applications (Lund, 1986), Lecture Notes in Math. 1302, Springer, Berlin, 1988, 158-170.

[5] E.W. Farkas, Function spaces of generalized smoothness and pseudo-differential operators associated to a continuous negative definite function, Habilitationschrift, LudwigMaximilian Universität München, 2002.

[6] E.W. Farkas and H.-G. Leopold, Characterisations of function spaces of generalised smoothness, Ann. Mat. Pura Appl. (4) 185 (2006), 1-62.

[7] W. Farkas and N. Jacob, Sobolev spaces on non-smooth domains and Dirichlet forms related to subordinate reflecting diffusions, Math. Nachr. 224 (2001), 75-104.

[8] W. Farkas, N. Jacob and R. Schilling, Feller semigroups, $L^{p}$-sub-Markovian semigroups, and applications to pseudo-differential operators with negative definite symbols, Forum Math. 13 (2001), 51-90.

[9] W. Farkas, N. Jacob and R. Schilling, Function spaces related to continuous negative definite functions: $\psi$-Bessel potential spaces, Dissertationes Math. (Rozprawy Mat.) 393 (2001), 62 pp.

[10] M. Fukushima, Y. Oshima and M. Takeda, Dirichlet forms and symmetric Markov processes, Walter de Gruyter \& Co., Berlin, 2011.

[11] N. Jacob, Feller semigroups, Dirichlet forms, and pseudodifferential operators, Forum Math. 4 (1992), 433-446.

[12] N. Jacob, A class of Feller semigroups generated by pseudo differential operators, Math. Z. 215 (1994), 151-166.

[13] N. Jacob, Pseudo-differential operators and Markov processes, Akademie-Verlag, Berlin, 1996. 
[14] N. Jacob, Pseudo-differential operators and Markov processes. Vol. I. Fourier analysis and semigroups, Imperial College Press, London, 2001.

[15] N. Jacob, Pseudo-differential operators and Markov processes. Vol. II. Generators and their potential theory, Imperial College Press, London, 2002.

[16] N. Jacob, Pseudo-differential operators and Markov processes. Vol. III. Markov processes and applications, Imperial College Press, London, 2005.

[17] N. Jacob and R. Schilling, Extended $L^{p}$ Dirichlet spaces, in: Around the research of Vladimir Maz'ya. I., Springer, New York, 2009, 221-238.

[18] A. Jonsson and H. Wallin, Function spaces on subsets of $\mathbb{R}^{n}$, Harwood Acad. Publ., 1984.

[19] P. Kim, R. Song, and Z. Vondraček, Martin boundary for some symmetric Lévy processes, Festschrift Masatoshi Fukushima, World Sci. Publ., Hackensack, 2015, 307-342.

[20] V. Knopova and M. Zähle, Spaces of generalized smoothness on $h$-sets and related Dirichlet forms, Studia Math. 174 (2006), 277-308.

[21] S.D. Moura, On some characterizations of Besov spaces of generalized smoothness, Math. Nachr. 280 (2007), 1190-1199.

[22] R. Schilling, R. Song and Z. Vondraček, Bernstein functions. Theory and applications, Walter de Gruyter \& Co., Berlin, 2009.

[23] I. J. Schoenberg, Metric spaces and completely monotone functions, Ann. of Math. (2) 39 (1938), 811-841.

[24] H. Triebel, Theory of function spaces. II, Birkhäuser, Basel, 1992.

[25] H. Triebel, Interpolation theory, function spaces, differential operators, North-Holland, Amsterdam-New York, 1978.

[26] V. Wagner, Censored symmetric Lévy-type processes, preprint, 2018, 20pp.

[27] H. Wallin, The trace to the boundary of Sobolev spaces on a snowflake, Manuscripta Math. 73 (1991), 117-125.

[28] M. Zähle, Potential spaces and traces of Lévy processes on h-sets, J. Contemp. Math. Anal. 44 (2009), 117-145.

Department of Mathematics

Faculty of Science

University of Zagreb

10000 Zagreb

Croatia

E-mail: wagner@math.hr

Received: 30.3 .2018$.

Revised: 17.10.2018. 